\title{
Cycling Changes in the Amplitudes of the 27-Day Variation of the Galactic Cosmic Ray Intensity
}

\author{
A. Gil - M.V. Alania
}

Received: 13 November 2010 / Accepted: 4 February 2012 / Published online: 6 March 2012

(C) The Author(s) 2012. This article is published with open access at Springerlink.com

\begin{abstract}
We study quasi-periodical changes in the amplitudes of the 27-day variation of the galactic cosmic ray (GCR) intensity, and the parameters of solar wind and solar activity. We have recently found quasi-periodicity of three to four Carrington rotation periods ( $3-4 \mathrm{CRP})$ in the amplitudes of the 27-day variation of the GCR intensity (Gil and Alania in J. Atmos. Solar-Terr. Phys. 73, 294, 2011). A similar recurrence is recognized in parameters of solar activity (sunspot number, solar radio flux) and solar wind (components of the interplanetary magnetic field, solar wind velocity). We believe that the 3-4 CRP periodicity, among other periodicities, observed in the amplitudes of the 27-day variation of the GCR intensity is caused by a specific cycling structure of the Sun's magnetic field, which may originate from the turbulent nature of the solar dynamo.
\end{abstract}

Keywords Cosmic rays · Solar dynamo · 27-days variation of the GCR intensity · Sun's differential roatation

\section{Introduction}

Various periodicities in the temporal changes of the galactic cosmic ray (GCR) intensity have been studied rather intensively up to the present time. We mention here only a few representative papers published in the last decade, e.g., Mursula, Zieger, and Vilppola (2003), Mavromichalaki et al. (2003), Ruzmaikin, Cadavid, and Lawrence (2008), Kudela (2009), Ma, Han, and Yin (2009), Chowdhury, Khan, and Ray (2010), Sabbah and Kudela (2011),

\footnotetext{
A. Gil (凶) · M.V. Alania

Institute of Mathematics and Physics, Siedlce University of Natural Science and Humanities, Siedlce,

Poland

e-mail: gila@uph.edu.pl

M.V. Alania

e-mail: alania@uph.edu.pl

M.V. Alania

Institute of Geophysics, Tbilisi State University, Tbilisi, Georgia
} 
and references therein. The periodicities of the GCR intensity at first approximation can be divided into three groups: long-, intermediate- (mid-), and short-term quasi-periodicities (see, e.g., Chowdhury, Khan, and Ray, 2010). We mainly study features of the mid-term periodicities ( $\sim 27$ days) connected with the Sun's rotation (e.g., Gil and Alania, 2001, 2010). Recently we found (Gil and Alania, 2011) a clear recurrence in the temporal changes of the amplitudes of the 27-day variation of the GCR intensity, and in parameters of solar activity (SA) and solar wind. Namely, we recognized a significant recurrence with a duration of three to four Carrington rotations (3-4 CRP) as a new type of quasi-periodic variation. We assume that the observed quasi-periodic variability with a 3-4 CRP recurrence is related to the cycling structure of the solar magnetic field resulting from the turbulent solar dynamo. Our purpose in this paper is to study features of the 3-4 CRP cycling of the 27-day variations of the GCR intensity, and the solar activity and solar wind parameters.

\section{Data and Methods}

We use the daily data of the Kiel neutron monitor (http://134.245.132.179/kiel/, from years 1958-2009), the solar wind velocity (SWV), strength and components $(B, B x, B y$, $B z$ ) of the interplanetary magnetic field (IMF; http://omniweb.gsfc.nasa.gov/, from 19732010), sunspot numbers (SSNs), and solar radio flux (SRF; http://spidr.ngdc.noaa.gov/spidr/, from 1958-2010). We also use the data of neutron monitors Potchefstroom, Oulu, and Rome in the period 1980-1981, and Moscow, Oulu, and Apatity in the period 19961998.

To reveal periodicities in the dataset, we use the power spectral analysis method. The power spectrum gives a view of the segment of a signal's power falling within given frequency domains (see, e.g., Press et al., 2002). It can be written in a simple form, as

$$
\Phi\left(\frac{2 \pi}{t}\right)=\left|\frac{1}{\sqrt{2 \pi}} \sum_{n=-2 T}^{2 T} f_{n} \cdot \mathrm{e}^{-\mathrm{i} \frac{2 \pi}{t} n}\right|^{2}=\frac{F\left(\frac{2 \pi}{t}\right) F\left(\frac{2 \pi}{t}\right)^{*}}{2 \pi},
$$

where $F\left(\frac{2 \pi}{t}\right)$ is the fast Fourier transform for discrete sampling function $f$ and i denotes an imaginary unit. But in practice we find periodicities using an estimator in the form (e.g., Gubbins, 2004)

$$
\Psi(\omega)=\sum_{n=-2 T}^{2 T} R_{n} W_{n} \cdot \mathrm{e}^{-\mathrm{i} \omega n}, \quad \omega=\frac{2 \pi}{t}, n=0, \pm 1, \ldots, \pm 2 T,
$$

where $R$ is an autocovariance and $W$ is a window function; in our calculation we use Parzen's window function (Kanasewich, 1981).

To find amplitudes of the 27-day variations of different parameters in this paper we use a harmonic analysis method (Gubbins, 2004):

$$
F(t)=\frac{a_{0}}{2}+\sum_{k=1}^{\infty}\left(a_{k} \cdot \cos \frac{2 \pi k t}{P}+b_{k} \cdot \sin \frac{2 \pi k t}{P}\right)=\sum_{k=1}^{\infty} A_{k} \cdot \sin \left(\frac{2 \pi k t}{P}+\varphi_{k}\right)
$$

where

$$
a_{0}=\frac{1}{p} \sum_{i=1}^{2 p} x_{i}, \quad a_{k}=\frac{1}{p} \sum_{i=1}^{2 p} x_{i} \cos \frac{\pi k i}{p}, \quad b_{k}=\frac{1}{p} \sum_{i=1}^{2 p} x_{i} \sin \frac{\pi k i}{p},
$$


Figure 1 Temporal changes of the amplitudes of the 27-day variation of the GCR intensity (A27 I [\%]) for Kiel neutron monitor during the period 1958-2009 (smoothed over 7 CR).

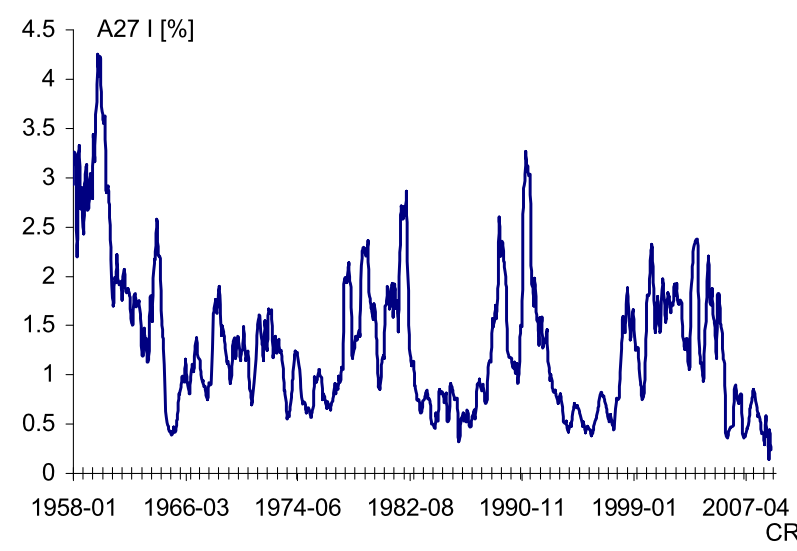

the period $P=2 p=27$ days, the amplitudes $A_{k}=\sqrt{a_{k}^{2}+b_{k}^{2}}$ of the 27-day variations of the GCR intensity, solar wind, and solar activity parameters (based on the daily data).

\section{Results and Discussion}

Figure 1 presents the temporal changes in the amplitudes of the 27-day variation of the GCR intensity by the Kiel neutron monitor for almost 700 Carrington rotations (1958-2009). Besides the clearly manifested 11-year variation of the amplitudes of the 27-day recurrence of the GCR intensity, Gil and Alania (2011) first recognized an existence of patterns with different cycling in the amplitudes of the 27-day variation of the GCR intensity. Among them a more clearly established one is the three to four Carrington rotation period $(3-4$ CRP); i.e., a clear recurrence with duration of 3-4 CRP was found. We continue to study the 3-4 CRP cycle using the data from various neutron monitors and other parameters of solar activity and solar wind.

Figures 2 and 3 show the 3-4 CRP cycling occurring in the amplitudes of the 27-day variations of different parameters: GCR intensity by neutron monitors Kiel, Potchefstroom, Oulu, and Rome (2a) and Kiel, Moscow, Oulu, and Apatity (3a), sunspot number (b), solar wind velocity (c), $B z$ component of IMF (d), and $10.7 \mathrm{~cm}$ solar radio flux (e) for the maximum (1980 - 1981; Figure 2) and minimum epochs (1996-1998; Figure 3) of solar activity. Figures 2 and 3 show a cycling of $3-4$ CRP in all considered parameters. Note that the cycling is clearest for the GCR intensity. Also, there is a significant correlation between cycling of the 3-4 CRP in the amplitudes of the 27-day variations of the GCR intensity and the solar wind velocity at minimum epochs of solar activity. However, these cyclic changes are sporadic phenomena; therefore, it is desirable to estimate the significance of the cyclicity.

Let us assume that there is no relationship between the possible 3-4 CRP features and the 27-day variation of the GCR intensity. In this case we can postulate that there exist different sources for the 3-4 CRP cycling structure and the 27-day variation of the GCR intensity; these two phenomena are independent and both are sporadic. We could observe the 3-4 CRP cycling only in the amplitudes of the 27-day variations of the GCR intensity and other parameters of solar activity and solar wind, but we have no direct observations of the 3-4 CRP cycling structure in the solar magnetic field. For both cases either the 27-day 
Figure 2 (a) - (e) Sequences of six of the 3-4 CRP cycling (1980 - 1981, maximum epoch of solar activity) of amplitudes of the 27-day variations of the: GCR intensity (A27 I [\%]) by Kiel, Oulu, Rome, and Potchefstroom neutron monitors (a), relative sunspot number (A27 of SSN, (b)), solar wind velocity (A27 of SWV, (c)), $B z$ component of IMF (A27 of $B z,(d))$, and $10.7 \mathrm{~cm}$ solar radio flux (A27 of SRF, (e)) with standard errors.

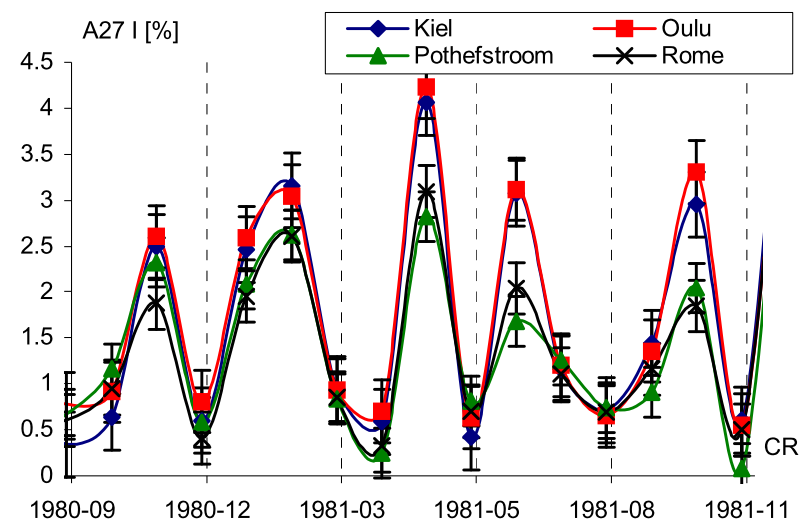

(a)

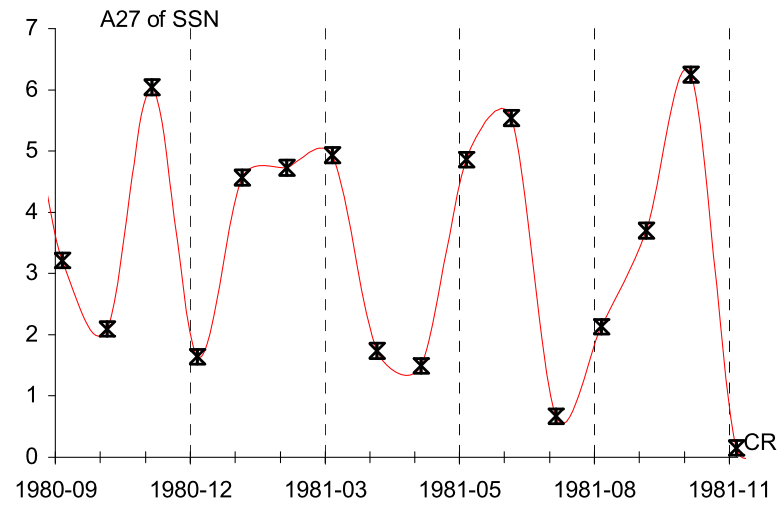

(b)

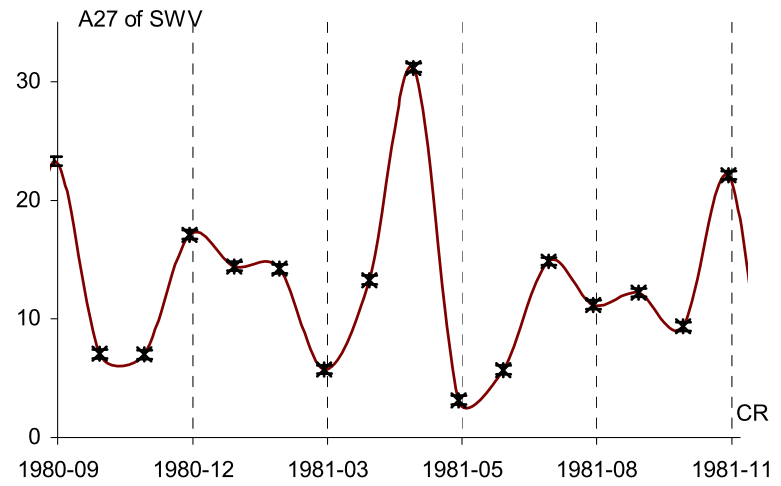

(c) 
Figure 2 (continued)

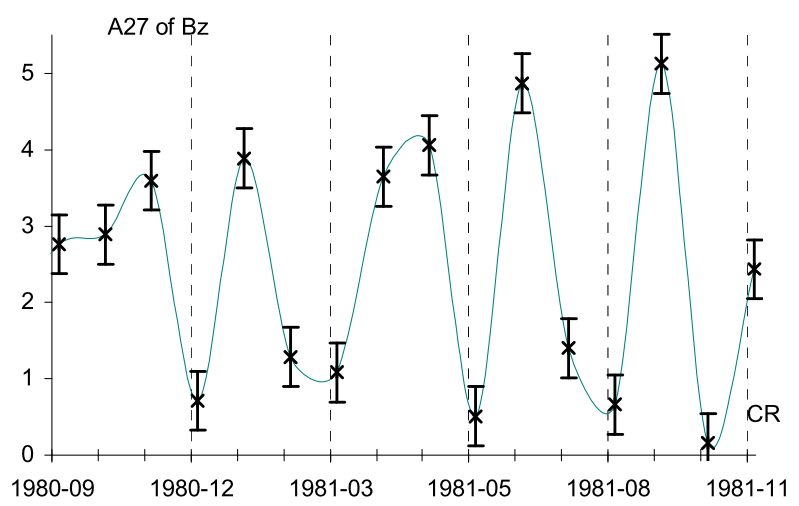

(d)

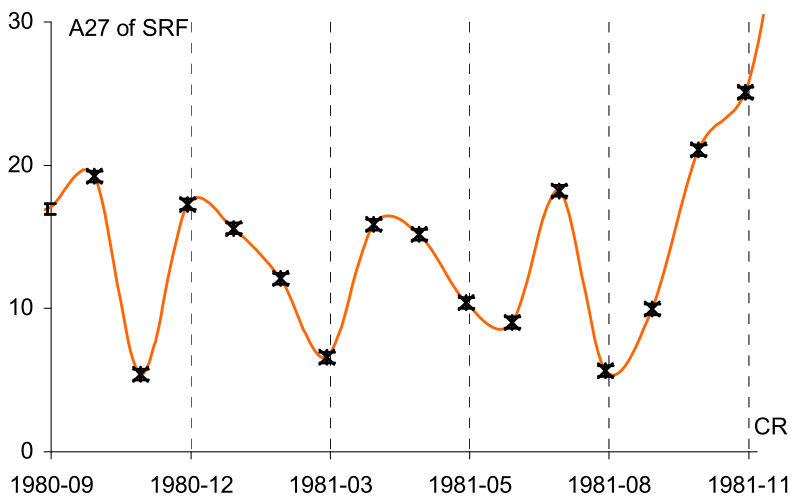

(e)

variation of the GCR intensity/or the 3-4 CRP cycling recognized in the 27-day variation of the GCR intensity is used as an indicator. However, we can compare the length of the time intervals without 3-4 CRP cyclic changes to those when recurrent changes are present. Let us consider the time interval 1973-2009, which is significantly longer than the duration of the 3-4 CRP and the 27-day variation of the GCR intensity. Taking into account that the 27-day variation of the GCR intensity (including some base values of its amplitude) exists for practically the whole period (at first approximation), the length of the time intervals with the 3-4 CRP cyclic changes in the amplitudes of the 27-day variation of the GCR intensity equals $\sim 46 \%$. Perhaps this feature can help us to better understand the processes taking place on the Sun.

We confirmed the occurrence of the 3-4 CRP cycling by calculating a power spectral density (PSD) of the amplitudes of the 27-day variations of the GCR intensity for the long period of 1958-2009 (Figure 4(a)) and of the GCR intensity and the solar wind velocity for the relatively short period of 1997-2009 (Figure 4(b), (c)). In both cases a periodicity of $\sim 3-4 \mathrm{CRP}$ is clearly noticeable.

As we have mentioned, the 3-4 CRP cycling has a rather sporadic character. It can exist for as long as two years, but after that it disappears, to emerge again after a few rotations. We suppose that the cycling is a universal property of solar activity connected with the 
Figure 3 (a) - (e) Sequences of six of the 3-4 CRP cycling (1996-1998, minimum epoch of solar activity) of amplitudes of the 27-day variations of the: GCR intensity (A27 I [\%]) by Kiel, Oulu, Moscow, and Apatity neutron monitors (a), relative sunspot number (A27 of

SSN, (b)), solar wind velocity (A27 of SWV, (c)), Bz component of IMF (A27 of $B z,(d))$, and $10.7 \mathrm{~cm}$ solar radio flux (A27 of SRF, (e)) with standard errors.

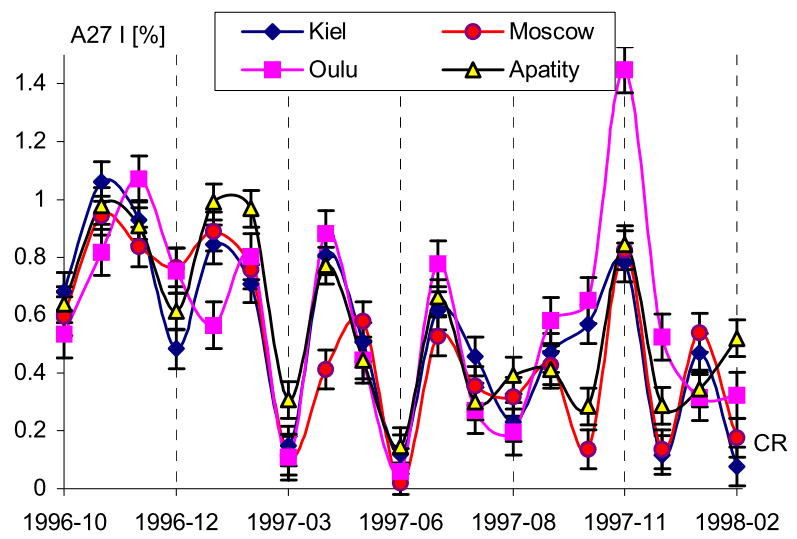

(a)

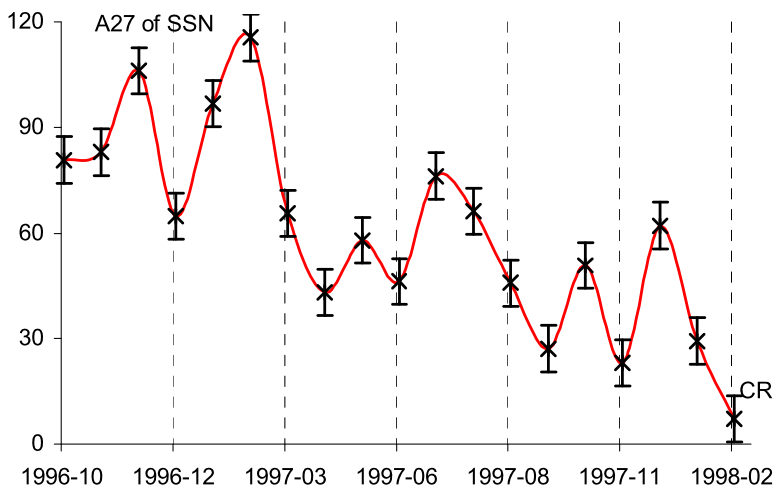

(b)

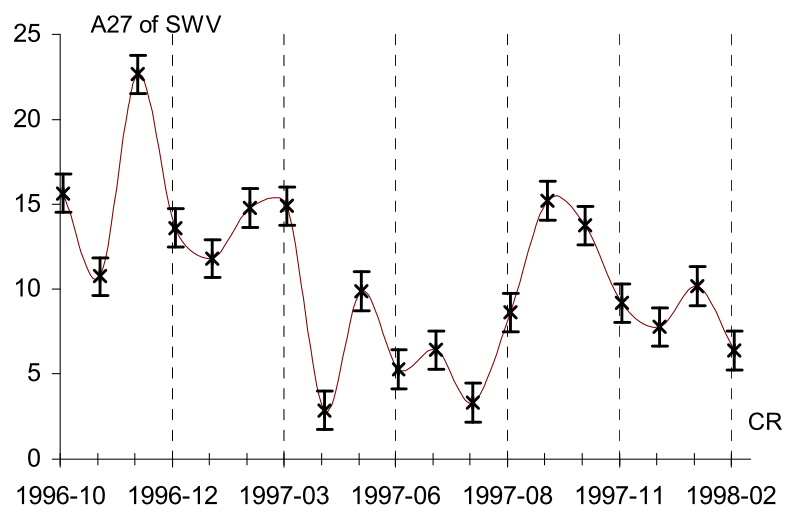

(c) 
Figure 3 (continued)

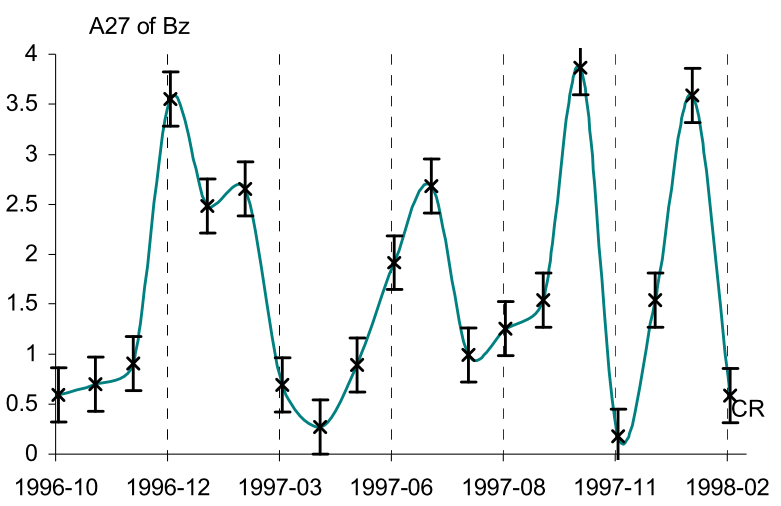

(d)

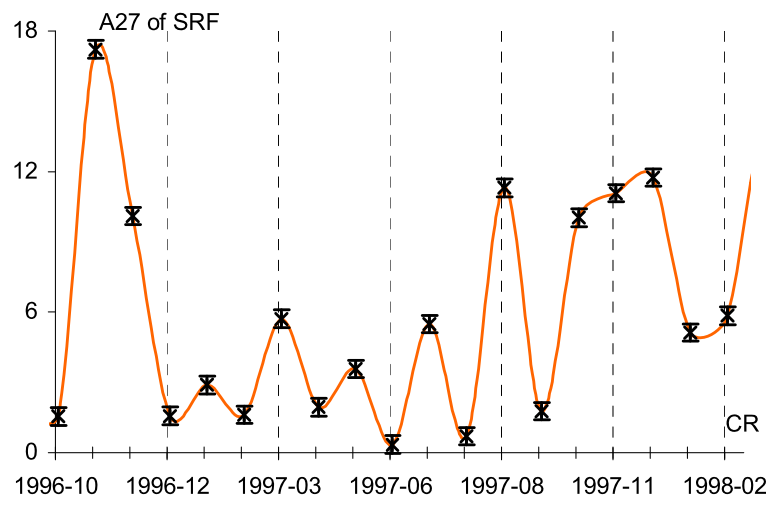

(e)

solar dynamo - sometimes disturbed by other processes, not very clearly visible, but still existing.

Generally, when proposing any new pattern (e.g., the 3-4 CRP structure of the magnetic field on the Sun), one must try to disprove a proposed model or to recommend other possible models. However, we could not find a more appropriate model. Thus, we accept a possible existence of the 3-4 CRP cycling structure of the Sun's magnetic field as a working hypothesis. In the temporal changes of the amplitudes of the 27-day variation of the GCR intensity, besides fluctuations of duration of 3-4 CRP, one clearly observes cycling structures with longer duration as well.

\section{Conclusions}

i) We identify a relatively clearly established quasi-recurrence with duration of three to four Carrington rotation periods $(3-4 \mathrm{CRP})$ in the temporal changes of the amplitudes of the 27-day variations of the GCR intensity, and parameters of solar activity (sunspot number, $10.7 \mathrm{~cm}$ solar radio flux) and solar wind (components of the interplanetary magnetic field, solar wind velocity). 
Figure 4 (a) - (c) PSD of the amplitudes of the 27-day variations of the GCR intensity $\left[(\%)^{2} \mathrm{~Hz}^{-1}\right]$ in the period 1958-2009 (PSD of A27 I, (a)) and in 1997-2009 (PSD of A27 I, (b)) and solar wind velocity $\left[\left(\mathrm{km} \mathrm{s}^{-1}\right)^{2} \mathrm{~Hz}^{-1}\right]$ in the period 1997-2009 (PSD of A27 SWV, (c)) with marked confidence level $99 \%$.

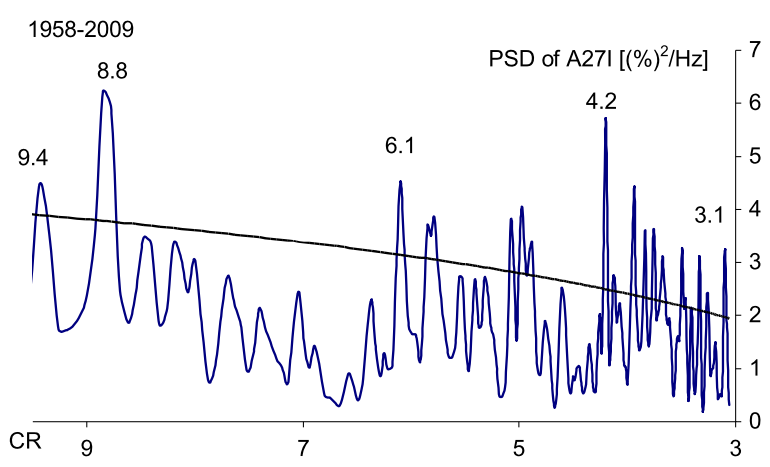

(a)

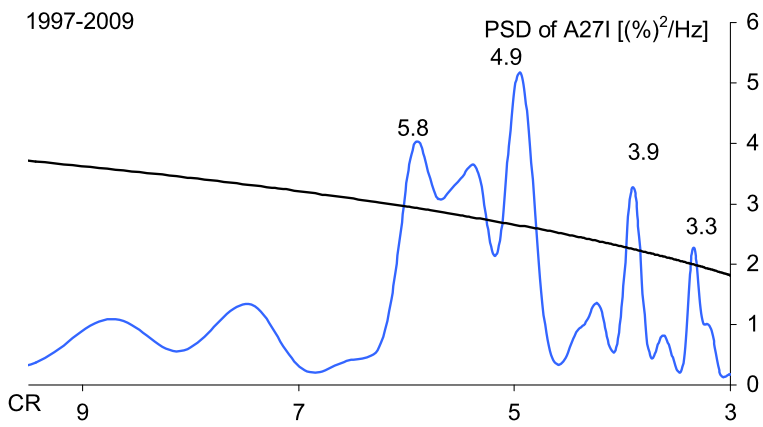

(b)

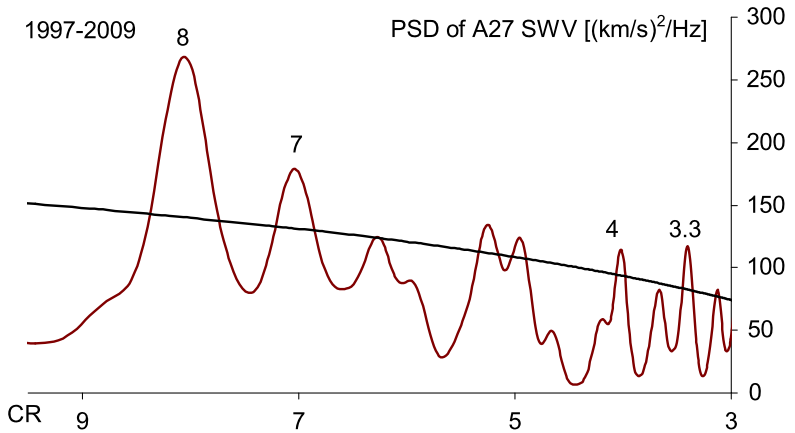

(c)

ii) We assume that the 3-4 CRP cycling structure is created by processes connected with the turbulent solar magnetic dynamo.

Acknowledgements The authors thank the referee and editors, who helped us to improve the paper; the investigators of the websites http://spidr.ngdc.noaa.gov/spidr/, http://omniweb.gsfc.nasa.gov/, and http://cro.izmiran.rssi.ru; and the researchers of the Kiel, Potchefstroom, Oulu, Apatity, Moscow, and Rome neutron monitors for allowing us to use their data. 
Open Access This article is distributed under the terms of the Creative Commons Attribution License which permits any use, distribution, and reproduction in any medium, provided the original author(s) and the source are credited.

\section{References}

Chowdhury, P., Khan, M., Ray, P.C.: 2010, Planet. Space Sci. 58, 1045.

Gil, A., Alania, M.V.: 2001, In: Proceedings of the 27th International Cosmic Ray Conference, Hamburg, Germany, 07-15 August, 2001. Under the auspices of the International Union of Pure and Applied Physics (IUPAP), 3725.

Gil, A., Alania, M.V.: 2010, Adv. Space Res. 45, 429.

Gil, A., Alania, M.V.: 2011, J. Atmos. Solar-Terr. Phys. 73, 294.

Gubbins, D.: 2004, Time Series Analysis and Inverse Theory for Geophysicists, Cambridge University Press, Cambridge, 21.

Kanasewich, E.R.: 1981, Time Sequence Analysis in Geophysics, The University of Alberta Press, Edmonton, 460 .

Kudela, K.: 2009, Acta Phys. Slovaca 59, 537.

Ma, L.H., Han, Y.B., Yin, Z.Q.: 2009, Solar Phys. 255, 187.

Mavromichalaki, H., Preka-Papadema, P., Petropoulos, B., Vassilaki, A., Tsagouri, I.: 2003, J. Atmos. SolarTerr. Phys. 65, 1021.

Mursula, K., Zieger, B., Vilppola, J.H.: 2003, Solar Phys. 212, 201.

Press, W.H., Teukolsky, S.A., Vetterling, W.T., Flannery, B.P.: 2002, The Art of Scientific Computing, Cambridge University Press, Cambridge, 550.

Ruzmaikin, A., Cadavid, A.C., Lawrence, J.: 2008, J. Atmos. Solar-Terr. Phys. 70, 2112.

Sabbah, I., Kudela, K.: 2011, J. Geophys. Res. 116, A04103. 